\title{
Characterization of Neutropenic Pediatric Patients sent to a referral centre
}

\author{
Gustavo Adolfo Lazo-Páez, Oscar Porras
}

\begin{abstract}
Aim: Neutropenia is a relatively common cause of patient referral to the Immunology and Pediatric Rheumatology Department of the National Children's Hospital. The present study characterizes the cases of neutropenia referred to this department between November 1988 and June 2008.

Methods: Eighty four patients between 0 and 12 years of age, were referred from November 6th, 1988 and June 1st, 2008. We performed a comprehensive descriptive analysis of the characteristics exhibited by these patients in terms of clinical course, pattern of infection, most common causative germs, complications and treatment applied.

Results: Neutropenia resolved spontaneously in $52.2 \%$ of the patients, and they were classified as transient neutropenia, $21.7 \%$ of the cases developed cyclic neutropenia, $13 \%$ of were categorized as benign chronic neutropenia, $7.2 \%$ developed severe chronic symptomatic neutropenia, $2.9 \%$ had neutropenia associated with type $1 \mathrm{~B}$ glycogenosis and $2.9 \%$ of the cases were not classifiable in any of the proposed categories. More than $50 \%$ of the cases were associated with an abnormal pattern of infection in terms of frequency, severity, multiplicity of systems involved, or the presence of opportunistic microorganisms. The upper respiratory tract was the most commonly affected system with infection. Prophylactic antibiotics were used in $39.1 \%$ of the cases and granulocyte colony stimulating factor was required in $11.6 \%$ at some stage of the course. The most frequently involved pathogens in infection were Pseudomonas aeruginosa, Staphylococcus sp and E. coli.

Conclusion: The vast majority of neutropenic patients had a benign clinical course. The same germs described in other series, caused infections in our patients.

The absolute neutrophil count at diagnosis has no effect on the incidence of recurrent infection, but the type or clinical course of neutropenia does.
\end{abstract}

Pediatric Immunology and Rheumatology Service, Hospital Nacional de Niños Dr. Carlos Sáenz Herrera

\section{Contact information:}

E-mail: gustavo.lazo@gmail.com
Keywords: neutropenia, granulocyte colony stimulation factor, recurrent infection, immunodeficienc, children.

Received: July $21^{\text {th }} 2009 \quad$ Accepted: December $8^{\text {th }} 2009$ 
Neutrophils are granulocytes with phagocytic activity, main participants of the immune system. As other types of leucocytes they are originated from the pluripotent stem cells in the bone marrow, under the influence of many other factors as stress, corticosteroid levels, complement fractions, catecholamines, cytokines (as IL-8), hematopoietic growth factors as the granulocyte colony stimulating factor (GCSF), and others.

The antimicrobial activity is due to their high potential for phagocytosis and intracellular lysis, enhanced by tissue migration activity (dependent on integrins) and microorganism's opsonisation by complement fractions or immunoglobulins. ${ }^{1}$

Circulating neutrophils are approximately $5 \%$ of the total, about $10 \%$ are adhered to the endothelium and the other $85 \%$ are located in the bone marrow. ${ }^{1-2}$

These cells have a short life span (about 6-12 hours) on peripheral blood, before they enter the tissues where they end a life cycle of up to 24 hours. $^{2}$

On these cell's cytoplasm there is a phenomenon called respiratory burst in which free radicals as hydrogen peroxide or hydrogen superoxide are generated. These free radicals with other substances as hypochlorous acid and chloride amides cause damage to the attacking agent's membrane destroying them. ${ }^{3}$

The neutrophils have other mechanisms of attack independent of oxygen and also have the capability of secreting pro-inflammatory cytokines that will help to avoid the establishment of infection and collaborate in the elimination of established infection, with the cooperation of the other components of the immune response. ${ }^{3}$

The different alterations on the neutrophil's activity might be a result of a lower number of available cells (neutropenia) or to defects on its antimicrobial activity.

Neutropenia is the result of one or more defects in the neutrophil's differentiation or proliferation on the bone marrow, or an increase on granulocyte peripheral destruction. ${ }^{4}$

Transitory neutropenia is frequently observed in the paediatric setting and is usually not longer than 3 weeks, lacking clinical relevance in the majority of cases. These transitory states are usually associated to viral infections during which either an alteration in myelopoiesis or a increase in granulocyte consumption may happen. There are many other causes of neutropenia, according to duration and the clinical presentation associated, making it necessary to individualize each case and to use the clinical and laboratory tools available to identify the etiology. ${ }^{4}$

The term "chronic neutropenia" is reserved for the presence of neutrophil count abnormally low for 6 or more months. ${ }^{5-6}$

The available scientific data states that the majority of children with chronic benign neutropenia have autoimmunity as an etiology, although in some cases the demonstration of antibodies is too difficult. ${ }^{6}$

The risk of infection in patients with chronic neutropenia is inversely related to the absolute neutrophil count (ANC) and the marrow's neutrophil reserve. ${ }^{7-8}$ The infectious agents more frequently found in neutropenic patients are part of the normal endogenous flora: Staphylococcus spp., Serratia marcescens, Klebsiella spp., other gram negatives and Aspergillus spp. ${ }^{9}$

The clinical compromise in these patients is usually more important on the airway and mucosa ( gingivitis, stomatitis, periodontitis rectal abscess, pneumonia and otitis media). 
Usually the risk of severe or invasive infection is increased in the group of patients with less than 500 absolute neutrophils per $\mathrm{mm}^{3}$.

Children with persistent symptomatic neutropenia may need further studies such as bone marrow aspirate, human immunodeficiency virus (HIV), serum immunoglobulins, and anti-neutrophilic antibodies, making this group of patients the ones requiring a closer follow-up in the Immunology Department. ${ }^{7}$

The clinical course is variable and goes from infectious complications that appear early and could threaten life to the benign course of

\section{Methods}

This study is part of the investigation "Epidemiology of Primary Immunodeficiencies in children and teenagers in control at the PIRD$\mathrm{NCH}$ ", approved by the Bioethical Committee of the National Children's Hospital "Dr. Carlos Saenz Herrera" (NCH) by the office CLOBI003-2006.

This is a retrospective, descriptive study with the following inclusion criteria: (1) children from $0-18$ years old referred to the $\mathrm{NCH}$ with the diagnosis of neutropenia in between November 6, 1988 and June 1, 2008. (2) two consecutive blood counts with at least 24 hours in between with ANC less than 1500 cells/mm3. (3) Having controls at the $\mathrm{NCH}$ for at least 6 months. Patients with neutropenia secondary to chemotherapy, bone marrow transplant, macrophage activation syndrome or infected with HIV were excluded from the study as also were excluded the patients with an incidental neutropenia during a haematological check up.

The Paediatric Immunology and Rheumatology Department of The National Children's Hospital "Dr. Carlos Saenz Herrera" (PIRD-NCH), receives references for neutropenia study from the whole country since 1985. Before this the patients with neutropenia were evaluated by the Haematology and General Paediatrics Department (Diagnostic centre) of this same hospital.

This study aims to characterize the neutropenic paediatric patient referred to the PIRD-NCH between November 1988 and June 2008.

incomplete medical records for adequate data collection.

Data was collected from medical registry and laboratory registry from the $\mathrm{NCH}$. Information was included in a data recollection sheet, including: place of origin, age, presence of a recurrent, abnormally severe, multisystem or opportunistic infection pattern; hospitalizations, haematological characteristics, nutritional status, family history and complications during the illness. Information was transferred to a data base and then analyzed with the program Statistica 6.0 from StatSoft company.

A descriptive global analysis was made. The categorical variables were expressed as frequencies and percentages while the continual were expressed as a median with intervals. The Chi square test was used to compare the proportions and percentages, the $t$ student test to compare two averages, the multiple regression analysis for statistical correlations between variables and single way ANOVA for multiple comparison, considering a $\mathrm{p}$ value of less than 0.05 to be of significance. 


\section{Results}

Eighty four cases were studied, 69 filled the inclusion criteria. The male: female rate was of 1.2 and there was no statistically significant difference on the frequency of neutropenia according to patient's sex $(p=0.80)$. The average age was of 2.7 years at the time of diagnosis, ranging from 0 to 11.8 years, higher than that found by Quintero on 2003 (Unpublished postgraduate thesis), who noted an average age of one year. There was no statistically significant relation between the type of neutropenia and the age at diagnosis $(p=0.29)$. The distribution of patients according to age and sex is on Table1.

\begin{tabular}{|c|c|c|c|c|}
\hline \multicolumn{5}{|c|}{$\begin{array}{l}\text { Table 1: Patients with Neutropenia seen at the PIRD-NCH, according to } \\
\text { average age and sex by type. November } 1988 \text { to June } 2008 \text {. }\end{array}$} \\
\hline Type & n $(\%)$ & Male & Female & $\begin{array}{c}\text { Average age at } \\
\text { diagnosis }\end{array}$ \\
\hline Transitory & $36(52.2)$ & 21 & 15 & 1.9 \\
\hline Cyclic & $15(21.7)$ & 6 & 9 & 3.6 \\
\hline Chronic benign & $9(13.0)$ & 7 & 2 & 4.1 \\
\hline Chronic severe & $5(7.2)$ & 2 & 3 & 3.4 \\
\hline Glycogenosis & $2(2.9)$ & 1 & 1 & 1.2 \\
\hline Unclassified & $2(2.9)$ & 1 & 1 & 2.9 \\
\hline TOTAL & $69(100)$ & 37 & 31 & 2.7 \\
\hline
\end{tabular}

The majority of patients studied are from San Jose, followed by Alajuela, Heredia, Puntarenas, Limon and Cartago. There were no patients coming from Guanacaste. The distribution of the rates for 100.000 habitants are on Table 2.

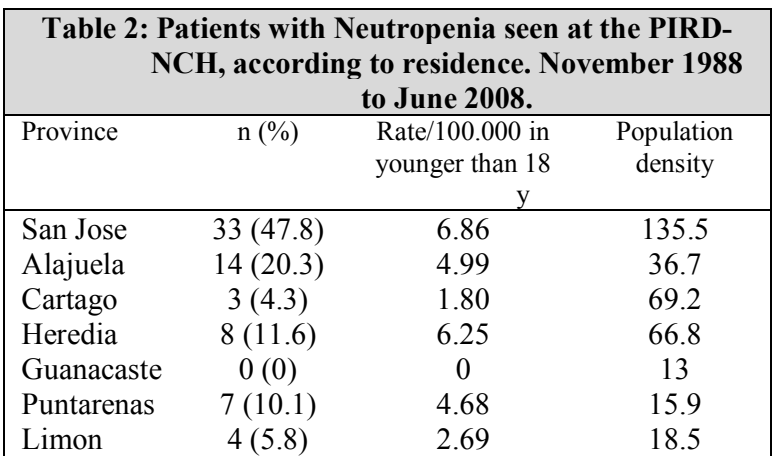

San Jose, Heredia and Cartago have the larger amount of patients, and these are the regions with the greatest population density. Population density habitants/ km2.

the PIRD-NCH for incidental neutropenia, 39.1\% were referred for neutropenia and an abnormal infection pattern (defined as recurrent, multisystem, unusually severe or due to opportunists type of infection). Only $5.8 \%$ of the cases were referred for an abnormal pattern of infection without neutropenia being documented previous to the evaluation at the PIRD-NCH.

In $92.8 \%$ of patients there was no familial history of immunodeficiencies or relevant perinatal history. It is probable that high blood pressure during pregnancy, which is a well studied event in relation to transient neutropenia in the newborn, was underestimated in this study due to the fact that it was not always present in the medical registry of the studied patients. ${ }^{1,3}$ Only in one patient there was history of chronic neutropenia in his father.

In $52.2 \%$ of patients spontaneous recovery of neutropenia was observed, these patients were assigned to the transitory neutropenia group. In this group of patients only one case there was need to use GCSF during a hospitalization. In $21.7 \%$ of cases the evolution was to cyclic neutropenia (one of these patients needing chronic administration of GCSF because of severe and recurrent infections), $13 \%$ of cases were assigned to the chronic benign neutropenia (none of them has needed the use of 


\begin{tabular}{|cccc|}
\hline \multicolumn{4}{|c|}{ Table 3: Patients with neutropenia seen at the PIRD/NCH, according to haematological } \\
characteristics. November 1988 to June 2008. \\
\hline Type & $\begin{array}{c}\text { Average } \\
\text { haemoglobin } \\
(\mathrm{g} / \mathrm{dL})\end{array}$ & $\begin{array}{c}\text { Average leucocytes } \\
\text { (cells/mm3) }\end{array}$ & $\begin{array}{c}\text { Average ANC (cells/mm3) } \\
\text { (c) }\end{array}$ \\
\hline Transitory & $11.6(9-15.5)$ & $6632(2660-20700)$ & $666(0-2280)$ \\
Cyclic & $11.9(9.2-13.9)$ & $5881(3280-9710)$ & $519(0-1004)$ \\
Chronic Benign & $12.3(9.9-14.4)$ & $4586(3100-6660)$ & $567(60-1076)$ \\
Chronic Severe & $10.3(8.4-12.3)$ & $5022(2430-8780)$ & $318(220-439)$ \\
Glycogenosis & $10.1(9.9-10.3)$ & $6900(3800-10000)$ & $550(400-700)$ \\
Unclassified & $11.7(11.7-11.7)$ & $3930(3300-4560)$ & $245(90-400)$ \\
\hline TOTAL & $11.6(8.4-15.5)$ & $6028(2430-20700)$ & $582(0-2280)$ \\
\hline
\end{tabular}

GCSF), $7.2 \%$ of cases the evolution was to chronic severe symptomatic neutropenia (three requiring the use of GCSF at some point), $2.9 \%$ of patients present neutropenia in association to glycogenosis type 1B, and all of them have used GCSF at some point, intermittent and for short periods of time. The $2.9 \%$ of cases were not classified in these groups. For each of these groups the hematologic characteristics and clinical presentation are summarized on Tables 3 and 4.

In $56.5 \%$ of patients there was an abnormal pattern of infection in association to the neutropenia, with recurrent infection affecting $84.6 \%$ of patients. In this study $67.9 \%$ of recurrent infections were upper respiratory infections, including acute otitis media, pharyngitis and acute tonsillitis. In $15 \%$ of patients with recurrent infection diarrhea was present, the same percentage was observed for skin infections (either abscess or recurrent pyodermitis). Only in one patient there was recurrent urinary tract infection, without any anatomical abnormality.

From the total of patients in the group of abnormal infection $12.8 \%$ of cases presented with "more severe than usual infection". In this group there was a patient with necrotic abscessed pneumonia that needed resection of the compromised lobe and decortication, a severe chicken pox with Pseudomona aeruginosa infection, one acute osteomyelitis of the tibia, one purulent tracheobronchitis, one abscess of the scalp with secondary osteomyelitis, one septicaemia due to
Staphylococcus haemolyticus, one axillar abscess with surgical drainage, three chronic otitis media with mastoiditis also surgically drained and one abscessed stomatitis that required hospitalization.

In the whole study there was only one infection due to an opportunistic agent in a girl with oesophageal candidiasis in an upper endoscopy without culture or microbiological analysis.

The frequency of patients with an abnormal pattern of infection was higher in the patients with transitory neutropenia and cyclic neutropenia (16 and 10 cases) and is illustrated on Table 4.

It was not possible to demonstrate a significant relationship between the type of neutropenia and the absolute neutrophil count at the time of diagnosis $(\mathrm{p}=0.44)$.

The more frequent germs identified as the cause of severe infection in these patients (as described on Table 5) are in order of frequency: Pseudomona aeruginosa, Staphylococcus spp., Escherichia coli, Salmonella B., and Varicella zoster virus.

It is important to point out that although there is an abnormal pattern of infection in neutropenic patients, only 12 germs were isolated, which is in correlation to the number of hospitalizations required for infection (associated to neutropenia), for the number of analyzed patients, that was of 24 in 18 patients, for a total of 5 hospitalizations for 100 patients-year (Table 5). 
The pattern of infection in neutropenic patients motivated the use of prophylactic antibiotics in $39.1 \%$ of patients and use of GCSF in $11.6 \%$. The type of neutropenia in these patients was not significantly associated to the decision to use prophylactic antibiotics $(\mathrm{p}=0.32)$. The most frequently used antibiotics were Trimethoprimsulfamethoxazole and Amoxicillin. The type of neutropenia was statistically significant associated to the use of GCSF ( $\square \square 0.001$ ), been more used in chronic severe neutropenia and glycogenosis as shown on Table 5. All patients that received GCSF as part of their treatment remained free of infection for the rest of the treatment.

Gingivitis, diarrhea and recurrent abscess were present in $5.8 \%, 5.8 \%$ and $13 \%$ of patients respectively, at some point of their evolution. It could only be demonstrated to be a relation between the type of neutropenia and the presence of gingivitis ( $\mathrm{p}=0.04)$ and abscess $(\mathrm{p} \square 0.001)$.

When the nutritional status of the patients was evaluated $85.5 \%$ of patients were eutrophic at the time of diagnosis, without significant changes at one year follow-up (Table 1).

In $69.6 \%$ of patients a bone marrow sample was not taken. In the group of patients with a bone marrow sample only one was abnormal (failure to mature in the granulocytic line). The association of neutropenia with other haematological abnormalities was rare. There was one case of pancytopenia, one thalassaemia and one iron-refractory anaemia without specific diagnosis.

In $68.1 \%$ of patients a quantitative immunoglobulin test was performed and one case of hypogammaglobulinaemia was found that has been unclassified to this date. There were no deaths in the patients studied.

\section{Discussion}

Patients with neutropenia are not only a diagnostic challenge, in many cases presenting etiologies requiring urgent treatment, but also a challenge on management and follow up, due to the lack of predictive factors on the clinical course as of which of them will be benign and which will require pharmacological interventions for severe infections.10-14

This study did not include the total of cases of neutropenia as some of these patients might be in control by community paediatricians (especially the ones without relevant clinical manifestations), could be in the Haematology Department of the NCH or other Departments at adult's hospitals if diagnosis was made after 13 years of age.

San Jose was the city with the largest amount of patients, probably due to the centralization of primary attending services at the country's capital, as the access to a paediatrician might be limited on more rural areas. Also, San Jose has the highest population density so it is expected to be the province with more cases in the study.

This study shows that patients with neutropenia are referred to the specialized consult later than was described by Quintero 5 years ago (unpublished postgraduate thesis). This suggests: under diagnosis, a better care at primary attention centres, better attention of infectious complications or a difficult access to specialized medicine. It is possible that families might have more than one neutropenic member, but due to its benign course the statistics are biased by the absence of symptoms.

When the impact of neutropenia on infection was analyzed it is important to considerate that patients cannot be outlined from their social environment as it has been widely demonstrated that the pattern of infection depends not only on the host factors but also its surroundings. 8 It would be of high 
interest to analyze the group of patients with an abnormal pattern of infection considering other factors as allergies, smoking exposure, home environment, attendance to day care centres, etc., that were not analyzed in this study.

The lack of positive results on cultures might suppose that the infections present in the symptomatic patients were common childhood infections. It is also possible that the sensitivity of the microbiological study might be higher if more samples are taken, which is necessary not only to choose a better treatment but also to identify the microorganisms to choose prophylactic antibiotics in the patients that may need them. ${ }^{16-20}$

The use of GCSF in patients with chronic severe neutropenia has demonstrated to significantly improve survival by lowering the frequency and severity of infection by stimulating proliferation and maturation of granulocytes and improving phagocytosis and increasing phagocyte's bactericide activity. $^{21}$

As neutrophils are important in keeping skins' and mucosal integrity it calls our attention the low frequency of gingivitis and recurrent abscess in this series of cases, although there is a clear statistically significant relation between sustained neutropenia and these symptoms as described in the medical literature. ${ }^{9,16}$

Some reviews as the one by Bernini in 1996, suggest that the bone marrow aspirate could be required as frequently as the immunoglobulin determination, especially in chronic neutropenia. 2

The management of patients with neutropenia can be done in two phases: an initial phase about establishing the evolution and pattern of neutropenia (sustained or cyclic, acute or chronic), and a second phase to evaluate the clinical manifestations of neutropenia in the patients evolution an time. On those patients with symptomatic persistent neutropenia the wide differential diagnosis makes it necessary to evaluate the bone marrow's reserve and correlate the granulocyte deficiency with other defects of the immune system or infections as HIV ( that can be associated to neutropenia in up to $75 \%$ of cases), 17 before life threatening complications appear, secondary to neutropenia etiology. ${ }^{22-24}$

The study was limited due to the small size of the sample, which is expected for a infrequent pathology with a benign course. Also it was necessary to exclude a big number of patients due to incomplete data recollection.

Special Thanks: This study would not have been possible without the help and support from the medical personnel of the PIRD-NCH, that is daily working for the necessities of these patients and fills the registry of data recollection, or without the secretaries of the PIRD-NCH that strengthen the Department's data base. 


\begin{tabular}{|c|c|c|c|c|}
\hline Type & $\begin{array}{c}\text { Abnormal pattern of } \\
\text { infection }\end{array}$ & Gingivitis & Diarrhoea & Abscess \\
\hline Transitory & 16 & 0 & 2 & 1 \\
\hline Cyclic & 10 & 1 & 1 & 2 \\
\hline Chronic benign & 5 & 1 & 0 & 1 \\
\hline Chronic severe & 4 & 2 & 0 & 4 \\
\hline Glycogenosis & 2 & 0 & 1 & 1 \\
\hline Unclassified & 2 & 0 & 0 & 0 \\
\hline TOTAL & 39 & 4 & 4 & 9 \\
\hline
\end{tabular}

\begin{tabular}{|c|c|c|c|}
\hline Type & Hospitalizations & GCSF & $\begin{array}{l}\text { Antibiotic } \\
\text { prophylaxis }\end{array}$ \\
\hline Transitory & 8 & 1 & 13 \\
\hline Cyclic & 8 & 1 & 4 \\
\hline Chronic benign & 2 & 0 & 2 \\
\hline Chronic severe & 9 & 3 & 5 \\
\hline Glycogenosis & 3 & 2 & 1 \\
\hline Unclassified & 1 & 1 & 2 \\
\hline TOTAL & 31 & 8 & 27 \\
\hline
\end{tabular}

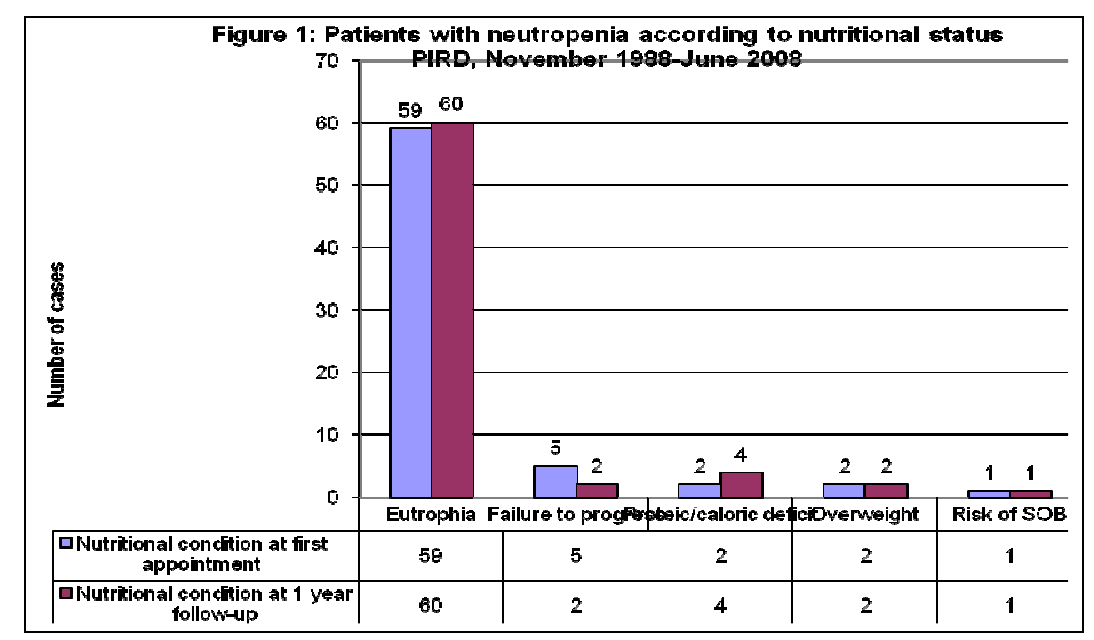




\section{References}

1. Lakshman R, Finn A. Neutrophil disorders and their management. J Clin Pathol. 2001; 54:7-19.

2. Bernini JC. Diagnosis and management of chronic neutropenia during childhood. Pediatr Clin North Am.1996; 43: 773-792.

3. Berliner N, Horwitz M, Loughran T. Congenital and adquired neutropenia. Hematology. 2004: 6379.

4. Boxer LA, Blackwood RA. Leukocyte disorders: Quantitative and qualitative disorders of the neutrophil, Part 2. Pediatr Rev. 1996; 17: 4751 .

5. James RM, Kinsey SE. The investigation and management of chronic neutropenia in children. Arch Dis Child. 2006; 91: 852-858.

6. Capsoni F, Sarzi-Puttini P, Zanella A. Primary and secondary autoimmune neutropenia. Arthritis Res Ther. 2005; 7: 208-214.

7. Horwitz M, Duan Z, Korkmaz B, Lee HH, Meallife ME, Salipante SJ. Neutrophil elastase in cyclic and severe congenital neutropenia. Blood. 2007; 109: 1817-1824.

8. Slatter MA, Gennery AR. Clinical Immunology Review Series: An approach to the patient with recurrent infections in childhood. Clin Exp Immunol. 2008; 152: 389-396.

9. Rosensweig S, Holland S. Phagocyte immunodeficiencies and their infections. J Allergy Clin Immunol. 2004; 113: 620-626.

10. Roskos RR, Boxer LA. Clinical disorders of neutropenia. Pediatr Rev. 1991;12: 208-213.

11. Dale D, Person RE, Bolyard AA, Aprikyan AG, Bos C, Bonilla MA et al. Mutations in the gene encoding neurophil elastase in congenital and cyclic neutropenia. Blood. 2000; 93: 2317-2322.

12. Online Mendelian Inheritance in Man. Elastase 2. Consultado el 26 de agosto de 2008 En: http://www.ncbi.nlm.nih.gov/entrez/dispomim. cgi?id=130130

13. Ancliff PJ. Congenital neutropenia. Blood Rev 2003; 17: 209-216.

14. Klein C, Grudzien M, Appaswamy G, Germeshausen M, Sandrock I, Schäffer AA et al. HAX1 deficiency causes autosomal recessive severe congenital neutropenia (Kostmann disease). Nat Genet. 2007; 39: 8692.

15. Boxer LA. Neutrophil abnormalities. Pediatr Rev. 2008; 24: 52-61.

16. Zaromb A, Chamberlain D, Schoor R, Almas K, Blei F. Periodontitis as a manifestation of chronic benign neutropenia. J Periodontol. 2006; 77:1921-1926.

17. Kuritzkes DR. Neutropenia, neutrophil dysfunction, and bacterial infection in patients with human immunodeficiency virus disease: the role of granulocyte colony-stimulating factor. Clin Infect Dis. 2000; 30: 256-260.

18. Newburger PE. Disorders of neutrophil number and function. Hematology. 2006; 7: 104-110.

19. Segel GB, Halterman JS. Neutropenia in pediatric practice. Pediatr Rev. 2008; 29: 12-25.

20. Boxer LA, Blackwood RA. Leukocyte disorders: Quantitative and qualitative disorders of the neutrophil, Part 1. Pediatr Rev 1996; 17: 19-29.

21. Lehrnbecher T, Welte K. Haematopoietic growth factors in children with neutropenia. $\mathrm{Br} \mathrm{J}$ Haematol. 2002; 116: 28-56. 
22. Lima C, Paula E, Takahashi T, Saad ST, Lorand-Metze S, Costa FFl. Causes of incidental neutropenia in adulthood. Ann Hematol. 2006; 85:

705-709.

23. Palmblad J, Papadaki HA. Chronic idiopathic neutropenias and severe congenital neutropenia. Curr Opin Hematol. 2008; 15: 8-14.

24. Papadaki H, Eliopoulos A, Kosteas T, Gemetzic C, Damianaki A, Kontala H et al. Impaired granulocytopoiesis in patients with chronic idiopathic neutropenia is associated with increased apoptosis of bone marrow progenitor cells. Blood. 2003; 101: 2591-26

\section{Translated by: Marcela Porras}


